\title{
The prevalence of adverse cardiometabolic responses to exercise training with evidence- based practice is low
}

This article was published in the following Dove Press journal:

Diabetes, Metabolic Syndrome and Obesity: Targets and Therapy

29 January 2015

Number of times this article has been viewed

\section{Lance C Dalleck' \\ Gary P Van Guilder ${ }^{2}$ \\ Tara B Richardson' \\ Chantal A Vella ${ }^{3}$}

'Recreation, Exercise, and Sport Science Department, Western State Colorado University, Gunnison, CO, USA; ${ }^{2}$ Department of Health and Nutritional Sciences, South Dakota State University, Brookings, SD, USA; ${ }^{3}$ Department of Movement Sciences, WWAMI Medical Education Program, University of Idaho, Moscow, ID, USA

Correspondence: Lance C Dalleck Recreation, Exercise, and Sport Science Department, Western State Colorado University, $600 \mathrm{~N}$ Adams Street,

Gunnison, CO, 81230, USA

Tel + I 9709437132

Fax + I 9709437125

Email Idalleck@western.edu
Background: The purpose of this study was to determine the prevalence of individuals who experienced exercise-induced adverse cardiometabolic response (ACR), following an evidencebased, individualized, community exercise program.

Methods: Prevalence of ACR was retrospectively analyzed in 332 adults (190 women, 142 men) before and after a 14-week supervised community exercise program. ACR included an exercise training-induced increase in systolic blood pressure of $\geq 10 \mathrm{mmHg}$, increase in plasma triglycerides (TG) of $>37.0 \mathrm{mg} / \mathrm{dL}$ ( $\geq 0.42 \mathrm{mmol} / \mathrm{L}$ ), or decrease in high-density lipoprotein cholesterol (HDL-C) of $>4.0 \mathrm{mg} / \mathrm{dL}(0.12 \mathrm{mmol} / \mathrm{L})$. A second category of ACR was also defined - this was ACR that resulted in a metabolic syndrome component (ACR-risk) as a consequence of the adverse response.

Results: According to the above criteria, prevalence of ACR between baseline and post-program was systolic blood pressure (6.0\%), TG (3.6\%), and HDL-C (5.1\%). The prevalence of ACR-risk was elevated TG (3.2\%), impaired fasting blood glucose (2.7\%), low HDL-C (2.2\%), elevated waist circumference (1.3\%), and elevated blood pressure $(0.6 \%)$.

Conclusion: Evidence-based practice exercise programming may attenuate the prevalence of exercise training-induced ACR. Our findings provide important preliminary evidence needed for the vision of exercise prescription as a personalized form of preventative medicine to become a reality.

Keywords: evidence-based research, cardiovascular health, community-based research, metabolic health

\section{Introduction}

It is well accepted that regular exercise has positive effects on health outcomes related to cardiovascular morbidity and mortality. ${ }^{1}$ Emerging evidence suggests individual variability in exercise-induced changes in common cardiovascular disease (CVD) risk factors, with some individuals experiencing adverse responses (a response in an unfavorable direction) when exposed to regular exercise. ${ }^{2-4}$ With exercise being recognized as an effective treatment for many obesity-related conditions, ${ }^{3}$ understanding factors associated with adverse responses is of growing importance. To date, few studies have evaluated adverse responses to exercise and results are conflicting. ${ }^{2-5}$ In a recent study ${ }^{2}$ that combined data from six exercise intervention studies, it was reported that adverse responses in individual cardiovascular and metabolic (cardiometabolic) risk factors ranged from $8 \%$ to $13 \%$ in sedentary adults undergoing 4 to 6 months of aerobic exercise training. Additionally, Yates et $\mathrm{al}^{5}$ reported $41 \%$ of participants randomized to a pedometer-based intervention experienced at least one adverse response in cardiometabolic risk factors during the 12-month study. Interestingly, these authors 
reported that the odds of an adverse response were reduced in those participants who significantly increased physical activity during the study, suggesting that a progressive exercise prescription may be important in reducing adverse responses to exercise. In contrast, a study reported less than a $1 \%$ prevalence of adverse responses in heart failure patients attending cardiac rehabilitation; however, this study only investigated adverse responses in $\mathrm{VO}_{2}$ peak. ${ }^{4}$ Although these findings suggest that there may be individuals who experience adverse responses in cardiometabolic risk factors when engaging in exercise, it is important to identify whether those who experience adverse responses fall within the healthy range for a given risk factor. Whether the heterogeneity in response to exercise increases cardiometabolic risk in certain individuals is unknown but of growing importance. Further, whether an individualized, evidence-based exercise program results in a lower prevalence of adverse responders is currently unknown. Therefore, the purpose of this study was to determine the prevalence of individuals who experienced one or more adverse responses in cardiometabolic risk factors, following an evidence-based, individualized, community exercise program. We hypothesized that an individualized, evidencebased exercise program would result in a low prevalence of adverse responses in cardiometabolic risk factors. We also hypothesized that the majority of those who experience an adverse response for a given risk factor would not increase their risk stratification and would remain in the healthy range for that risk factor.

\section{Methods}

\section{Study population}

A cohort of 332 adults (190 women, 142 men; age range 28-88 years) from a prior study ${ }^{6}$ in which components of metabolic syndrome (MetS) were measured before and after a 14-week evidenced-based community exercise program were analyzed for adverse cardiometabolic responses in the present study. All participants underwent a complete medical history and health assessment to determine if it was safe to engage in regular exercise. Participants were excluded from the study if they presented with a history of cardiovascular, pulmonary, or metabolic disease. This study was approved by the Institutional Review Board for the Protection of Human Subjects at the University of Wisconsin-Eau Claire.

\section{Assessment of cardiometabolic abnormalities}

Body mass and height were measured using a medical beam balance (Cardinal Scale Manufacturing Co, Webb City,
MO, USA). Body mass index was calculated as weight (kilograms) divided by height (meters) squared. Abdominal obesity was estimated by waist circumference measured at the smallest part of the abdomen, at the highest part of the iliac crest at the end of normal expiration. Auscultatory resting blood pressure was measured twice on at least two separate occasions following 5 minutes of seated quiet rest using standard procedures. A small sample of blood was obtained from the index finger and analyzed for fasting plasma lipids, lipoproteins, and glucose concentrations under strict standardized operating procedures (Cholestech LDX System, Alere Inc., Waltham, MA, USA). Independent studies indicate that the Cholestech system has excellent reproducibility with standard clinical laboratory measurement of plasma lipids and lipoproteins ${ }^{7,8}$ and meets the National Cholesterol Education Program Adult Treatment Panel (NCEP-ATP) III criteria for accuracy and reproducibility. ${ }^{9}$ Conventional sub-maximal exercise tests (ie, Rockport walk protocol or the Astrand-Rhyming cycle ergometry protocol) were administered using standardized procedures ${ }^{10}$ to estimate cardiorespiratory fitness. The sub-maximal tests selected for each participant were based on their training goals and whether they presented with musculoskeletal limitations that precluded the safe use of a particular test. Each participant completed the same sub-maximal exercise test at baseline and after the exercise program.

\section{Evidence-based community exercise programming}

A complete description of the sample, study design, and exercise training protocol has been described elsewhere. ${ }^{6}$ Briefly, using evidence from the STRRIDE study, each participant was provided an exercise prescription with an individualized weekly energy expenditure goal of $14-23 \mathrm{kcal} \cdot \mathrm{kg}^{-1} \cdot$ week $^{-1} \cdot{ }^{11-13}$ The weekly energy expenditure goal was used to calculate a minimum weekly exercise duration necessary to modify cardiovascular risk (corresponding to $40 \%-60 \%$ target $\mathrm{VO}_{2}$ reserve) using the following calculation:

$$
\begin{aligned}
& \text { Minimum minutes of exercise per week } \\
& =\left[14 \mathrm{kcal} \cdot \mathrm{kg}^{-1} \cdot \text { week }^{-1} \times \text { participant body mass }\right] \\
& \quad \div\left[\left(40 \% \text { target } \mathrm{VO}_{2}\right.\right. \text { reserve work load } \\
& \left.\quad \times \text { participant body mass }) \div 1000 \times 5 \mathrm{kcal} \cdot \mathrm{L} \mathrm{O}_{2}^{-1}\right]
\end{aligned}
$$

Exercise volume (minutes/week) was then divided by the target frequency goal for each participant to obtain daily exercise duration. Appropriate and safe individualized exercise prescriptions were designed for each participant by an 
exercise physiologist and also based on differences in age, sex, and fitness. In general, exercise volume and exercise intensity were progressed according to published guidelines. ${ }^{10}$ Each participant consulted with a team of exercise professionals and was assigned a University of Wisconsin-Eau Claire Department of Kinesiology senior student who served as their exercise trainer. The trainers worked directly under the supervision of qualified $\mathrm{PhD}$-trained exercise physiologists. Exercise training consisted of brisk walking, water aerobics, stationary cycling or cross-training, and supervised strength training for at least 3 days per week between 5:30 am and 8 am. Trainers coached participants during exercise sessions, provided motivational support, engaged in spotting, and corrected exercise technique.

\section{Definition of adverse cardiometabolic response to exercise}

Two separate definitions of exercise-induced adverse cardiometabolic response (ACR) to exercise were established. Our first ACR definition used threshold criteria similar to those published elsewhere. ${ }^{2}$ These included an exercise training-induced increase in systolic blood pressure (SBP) of $\geq 10 \mathrm{mmHg}$, increase in plasma triglycerides (TG) of $>37.0 \mathrm{mg} / \mathrm{dL}$ ( $\geq 0.42 \mathrm{mmol} / \mathrm{L})$, or decrease in highdensity lipoprotein cholesterol (HDL-C) of $>4.0 \mathrm{mg} / \mathrm{dL}$ $(\geq 0.12 \mathrm{mmol} / \mathrm{L})$. A second definition of ACR was ACR that resulted in a net gain of one MetS component (ACR-risk) as a consequence of the adverse response. For example, an exercise training-induced increase of $12 \mathrm{mmHg}$ resulting in an SBP increase from $126 \mathrm{mmHg}$ to $138 \mathrm{mmHg}$ would transition an individual into the "positive" MetS component category for elevated blood pressure (ie, ACR-risk). The threshold criteria for MetS components were established according to NCEP-ATP III criteria. ${ }^{14,15}$ Framingham risk score, using the sex-specific algorithm for 10-year CVD risk, was calculated as previously described. ${ }^{16}$

\section{Statistical analysis}

All analyses were performed using Statistical Package for the Social Sciences, Version 19.0 (IBM Corporation, Armonk, NY, USA). All variables were initially checked for normality. Measures of centrality and spread are presented as frequency and mean \pm standard deviation. Fisher's exact chi-square $\left(\chi^{2}\right)$ tests were used to analyze the point prevalence of ACR and ACR-risk in the total cohort between baseline and postprogram. Mean differences in 10-year CVD risk between baseline and post-program for ACR-risk were assessed using paired $t$-tests. Mean differences in change in $\mathrm{VO}_{2} \max$ and post-program relative energy expenditure between those participants who did not experience an ACR "no ACR-risk" and "ACR-risk" were assessed using independent $t$-tests. The level of statistical significance was set at $P<0.05$ for all analyses.

\section{Results}

All participants completed the 14-week exercise program without incident, although there was variability in the volume of exercise performed. The mean exercise intensity for the cohort was $42 \% \mathrm{VO}_{2}$ reserve (range: $26 \%-76 \% \mathrm{VO}_{2}$ reserve).

\section{Prevalence of ACR}

Evidence-based practice exercise programming favorably impacted the cardiometabolic profile of many participants. Changes in physical and physiological characteristics of the study population in response to the exercise program have been reported elsewhere in a prior publication. ${ }^{6}$ When examining the prevalence of specific MetS risk factor components using $\chi^{2}$ difference testing, there were significant differences $(P<0.05)$ in all MetS risk factors components between baseline and post-program. In fact, the percentage of participants with elevated waist circumference (49.7\%), impaired fasting blood glucose $(30.1 \%)$, low HDL-C (21.4\%), elevated TG $(20.2 \%)$, and elevated blood pressure $(2.1 \%)$ was significantly $(P<0.05)$ reduced post-program.

When examining the prevalence of ACR using $\chi^{2}$ difference testing, there were non-significant differences $(P>0.05)$ between baseline and post-program. Overall prevalence of ACR was 4.9\% (49/996). The breakdown of each individual ACR was 6.0\% (20/332) for SBP, 3.6\% (12/332) for TG, and $5.1 \%$ (17/332) for HDL-C. The prevalence of multiple ACR was $1.2 \%$ (4/332). The distribution of cardiometabolic responses for SBP, HDL-C, and TG to the evidence-based community exercise program are presented in Figure 1.

\section{Prevalence of ACR-risk}

When examining the prevalence of ACR-risk using $\chi^{2}$ difference testing, there were non-significant differences $(P>0.05)$ between baseline and post-program. The prevalence of ACR-risk for elevated TG (3.2\%), impaired fasting blood glucose $(2.7 \%)$, low HDL-C $(2.2 \%)$, elevated waist circumference $(1.3 \%)$, and elevated blood pressure $(0.6 \%)$ was low. More men $(n=15)$ compared to women $(n=7)$ were ACR-risk. There was only a single individual with multiple ACR-risk. The breakdown of no ACR-risk and ACR-risk for each MetS component, along with training outcome markers, 

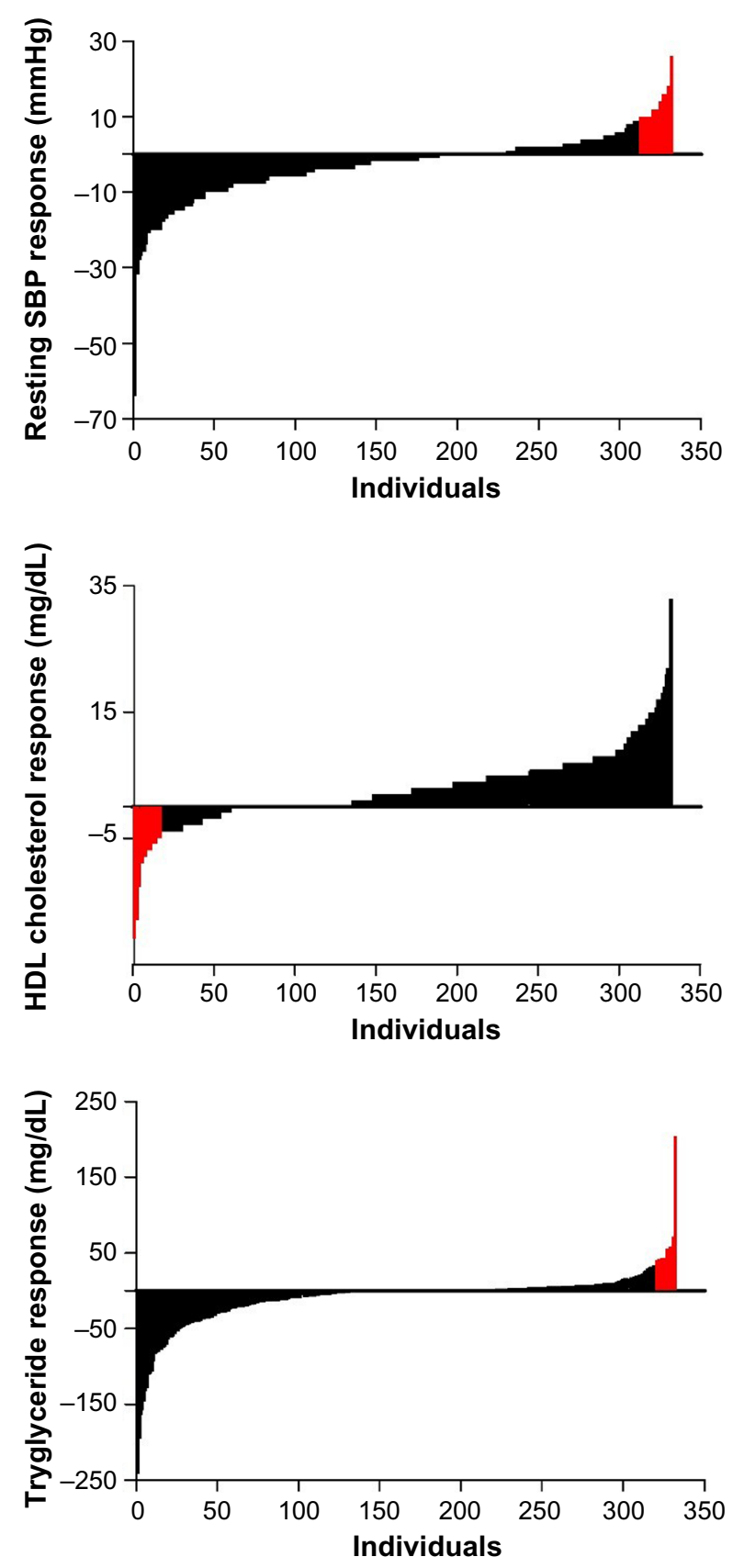

Figure I Distribution of all cardiometabolic responses for systolic blood pressure (SBP), high-density lipoprotein cholesterol (HDL-C), and triglyceride to the evidence-based community exercise program.

Note: Red color represents exercise training-induced ACR.

Abbreviation: ACR, exercise-induced adverse cardiometabolic response.

are presented in Table 1. Independent $t$-tests showed no significant differences $(P>0.05)$ for energy expenditure and change in cardiorespiratory fitness between the "no-ACR risk" and "ACR-risk" cohorts.

Demographics, 10-year CVD risk scores at baseline and post-program, and change in cardiorespiratory fitness for each individual who presented with ACR-risk are shown in Table 2. Paired $t$-tests revealed no significant mean
Table I Breakdown of no ACR-risk and ACR-risk for each MetS component and training outcome markers (energy expenditure and change in cardiorespiratory fitness) for each group

\begin{tabular}{lllc}
\hline $\begin{array}{l}\text { MetS } \\
\text { component }\end{array}$ & Group (n) & $\begin{array}{l}\text { EE } \\
\text { (kcal/kg/wk) }\end{array}$ & $\begin{array}{c}\text { Change CR } \\
\text { fitness (\%) }\end{array}$ \\
\hline Elevated WC & no ACR-risk (I52) & $18.2 \pm 6.3^{\mathrm{a}}$ & $9.8 \pm 10.8$ \\
& ACR-risk (2) & $15.5 \pm 2.0$ & $5.8 \pm 2.1$ \\
Elevated BP & no ACR-risk (307) & $17.2 \pm 5.8$ & $10.5 \pm 12.1$ \\
& ACR-risk (2) & $15.8 \pm 9.9$ & $11.4 \pm 9.6$ \\
Low HDL-C & no ACR-risk (228) & $16.6 \pm 5.0$ & $9.0 \pm 5.3$ \\
& ACR-risk (5) & $10.2 \pm 11.5$ & $8.0 \pm 9.0$ \\
Elevated TG & no ACR-risk (239) & $17.1 \pm 5.8$ & $10.3 \pm 11.5$ \\
& ACR-risk (8) & $18.3 \pm 4.8$ & $12.6 \pm 18.1$ \\
IFG & no ACR-risk (I77) & $17.0 \pm 6.6$ & $10.1 \pm 10.3$ \\
& ACR-risk (5) & $16.4 \pm 2.2$ & $7.9 \pm 4.5$ \\
\hline
\end{tabular}

Notes: ${ }^{a}$ Mean \pm standard deviation. ACR-risk: adverse cardiometabolic response who gained a metabolic syndrome component. No ACR-risk: no adverse cardiometabolic response.

Abbreviations: ACR, exercise-induced adverse cardiometabolic response; MetS, metabolic syndrome; EE, energy expenditure; CR, cardiorespiratory; WC, waist circumference; BP, blood pressure; HDL-C, high-density lipoprotein cholesterol; TG, triglycerides; IFG, impaired fasting blood glucose.

differences $(P>0.05)$ between baseline and post-program 10-year CVD risk scores.

\section{Discussion}

The primary finding of the present study was that prevalence of ACR was low amongst participants within an evidencebased, community exercise program. Indeed, the prevalence of ACR and ACR-risk ranged from $3.6 \%$ to $6.0 \%$ and $0.6 \%$ to $3.2 \%$, respectively. Moreover, our findings showed very few multiple exercise training-induced ACR (1.2\%). These ACR figures are substantially lower than those reported previously in the literature ${ }^{2}$ which ranged from $10.3 \%$ to $13.3 \%$, and support the tremendous potential of evidencebased exercise programming to enhance training efficacy and limit training unresponsiveness.

It is plausible that differences in prevalence of ACR between the present study and those reported elsewhere ${ }^{2}$ can be attributable to evidence-based practice. Evidence-based practice, as it pertains to exercise prescription, entails integration of the highest available level of research evidence, clinical exercise physiologist expertise, and patient/client needs. ${ }^{17,18}$ Randomized controlled trials address the first two aspects of evidence-based practice; however, patient/client needs are rarely considered as it compromises integrity of the research design. For example, participants in a study who are unable to complete the recommended weekly volume of exercise are not provided with the alternative of a reduced weekly goal. Similarly, if a participant finds the target exercise intensity less than desirable, it is not permissible for intensity 
Table 2 Demographics, 10-year CVD risk scores at baseline and post-program, and change in cardiorespiratory fitness for each individual ACR-risk

\begin{tabular}{|c|c|c|c|c|c|c|}
\hline $\mathbf{N}$ & $\begin{array}{l}\text { Age } \\
\text { (yrs) }\end{array}$ & Sex & $\begin{array}{l}\text { MetS } \\
\text { component (s) }\end{array}$ & $\begin{array}{l}\text { BL I0-yr } \\
\text { CVD risk (\%) }\end{array}$ & $\begin{array}{l}\text { PP I0-yr } \\
\text { CVD risk (\%) }\end{array}$ & $\begin{array}{l}\text { Change } \\
\text { CR fitness (\%) }\end{array}$ \\
\hline I & 55 & Men & Elevated WC & 4 & 3 & 4.3 \\
\hline 2 & 45 & Men & Elevated WC/BP* & 3 & 3 & 7.3 \\
\hline 3 & 62 & Men & Elevated BP & 10 & 9 & 18.3 \\
\hline 4 & 52 & Men & Elevated BP & 2 & 2 & 4.6 \\
\hline 5 & 79 & Women & Elevated TG & 2 & 2 & 12.6 \\
\hline 6 & 63 & Women & Elevated TG & 2 & 2 & -5.0 \\
\hline 7 & 63 & Men & Elevated TG & 11 & 13 & 20.8 \\
\hline 8 & 48 & Men & Elevated TG & 2 & 2 & 8.9 \\
\hline 9 & 55 & Men & Elevated TG & 6 & 4 & 22.2 \\
\hline 10 & 58 & Women & Elevated TG & 1 & I & 1.8 \\
\hline II & 46 & Men & Elevated TG & 4 & 4 & 13.6 \\
\hline 12 & 65 & Men & Elevated TG & 11 & 10 & -9.1 \\
\hline 13 & 51 & Men & Low HDL-C & 3 & 5 & 0.5 \\
\hline 14 & 55 & Men & Low HDL-C & 9 & 8 & 20.6 \\
\hline 15 & 63 & Women & Low HDL-C & 2 & 2 & 13.5 \\
\hline 16 & 77 & Men & Low HDL-C & 16 & 15 & 0.3 \\
\hline 17 & 57 & Men & Low HDL-C & 7 & 8 & 5.7 \\
\hline 18 & 79 & Men & IFG & 30 & 30 & 13.3 \\
\hline 19 & 61 & Women & IFG & 2 & 2 & 1.8 \\
\hline 20 & 56 & Women & IFG & 0 & 0 & 5.7 \\
\hline 21 & 63 & Women & IFG & 1 & 0 & II.I \\
\hline 22 & 45 & Men & IFG & 3 & 3 & 7.3 \\
\hline Mean & 59.0 & - & - & 6.0 & 5.8 & 8.2 \\
\hline SD & 10.0 & - & - & 6.8 & 6.8 & 8.3 \\
\hline
\end{tabular}

Notes: *Denotes multiple ACR-risk. ACR-risk: adverse cardiometabolic response who gained a metabolic syndrome component.

Abbreviations: ACR, exercise-induced adverse cardiometabolic response; MetS, metabolic syndrome; EE, energy expenditure; CR, cardiorespiratory; WC, waist circumference; BP, blood pressure; HDL-C, high-density lipoprotein cholesterol; TG, triglycerides; IFG, impaired fasting blood glucose; CVD, cardiovascular disease; yr, year; BL, baseline; PP, post-program; SD, standard deviation.

to be titrated upward to meet individual preferences. Yet in the clinical setting, it is paramount that exercise prescription design/implementation/progression includes input from the individual. Numerous factors, including differences in fitness, body weight, sex, and personal exercise preferences, all must be accounted for to improve the quality of the individual exercise prescription to favorably modify cardiometabolic risk.

It is well established that considerable interindividual variability occurs in the positive physiological adaptations to exercise training. ${ }^{19}$ However, only more recently has the phenomenon of ACR been comprehensively explored, ${ }^{2}$ and presently no standardized definition for ACR exists in the literature. Classification and interpretation of an ACR may require a holistic view that integrates all exercise training outcomes. For example, in the present study an ACR rarely resulted in higher risk. In fact, 10-year CVD risk increased in only three individuals as highlighted in Table 2. This finding has practical implications suggesting that although some individuals may have adverse cardiometabolic responses to exercise training, this may not always result in increased risk and exercise may benefit these participants in different ways, such as improved cardiorespiratory fitness. Indeed, more than $40 \%$ (9/22) of ACR-risk individuals concurrently increased cardiorespiratory fitness levels by $10 \%$ or more (see Table 2 ). Higher levels of cardiorespiratory fitness have been offered as an antidote toward other risk factors. Moreover, the literature suggests a $15 \%$ reduction in mortality for a $10 \%$ improvement in cardiorespiratory fitness. ${ }^{20}$ Taken together, it may be unintentionally misleading to categorize someone as an "adverse responder to exercise" based on a single cardiometabolic factor when it is well known that regular exercise training confers a myriad of benefits. Clearly, the topic of adverse responders to exercise warrants much additional study.

There are a few experimental considerations regarding the present study. First, as with all retrospective studies, we cannot rule out the possibility that other factors (eg, dietary and sedentary behavior/sitting time) contributed to prevalence of ACR, irrespective of the community exercise program. Second, we applied criteria for ACR established elsewhere rather than based on a quantification of our own technical error measures. Had we done the latter our ACR prevalence data may have differed. Third, our sample was 
a convenience sample of volunteers, which may limit the generalizability of the findings.

In conclusion, evidence-based practice exercise programming may attenuate the prevalence of exercise-induced ACR in adults. Our findings provide important preliminary evidence needed for the vision of exercise prescription as a personalized form of preventative medicine to become a reality. Indeed, for the "exercise is medicine" mantra to be fully realized, the same evidence-based practice approach widely applied in the medical community must too be embraced within the discipline of exercise science.

\section{Acknowledgments}

No financial support was provided to conduct this study. The authors would like to acknowledge all participants and students involved with the Community Fitness Program.

\section{Disclosure}

The authors declare that there are no conflicts of interest.

\section{References}

1. Warburton DE, Nicol CW, Bredin SS. Health benefits of physical activity: the evidence. CMAJ. 2006;174(6):801-809.

2. Bouchard C, Blair SN, Chruch TS, et al. Adverse metabolic response to regular exercise: is it a rare or common occurrence? PLoS ONE. 2012;7(5):e37887.

3. Buford TW, Roberts MD, Church TS. Toward exercise as personalized medicine. Sports Med. 2013;43(3):157-165.

4. Leifer ES, Brawner CA, Fleg JL, et al. Are there negative responders to exercise training among heart failure patients? Med Sci Sports Exerc. 2014;46(2):219-224.

5. Yates T, Davies MJ, Edwardson C, Bodicot DH, Biddle SJ, Khunti K. Adverse responses and physical activity: secondary analysis of the PREPARE trial. Med Sci Sport Exerc. 2014;46(8):1617-1623.

6. Dalleck LC, Van Guilder GP, Quinn EM, Bredle DL. Primary prevention of metabolic syndrome in the community using an evidence-based exercise program. Prev Med. 2013;57(4):392-395.

7. Dale RA, Jensen LH, Krantz MJ. Comparison of two point-of-care lipid analyzers for use in global cardiovascular risk assessments. Ann Pharmacother. 2008;42(5):633-639.
8. Shephard MD, Mazzachi BC, Shephard AK. Comparative performance of two point-of-care analysers for lipid testing. Clin Lab. 2007;53(9-12): 561-566.

9. Bachorik PS, Ross JW. National Cholesterol Education Program recommendations for measurement of low-density lipoprotein cholesterol: executive summary. The National Cholesterol Education Program Working Group on Lipoprotein Measurement. Clin Chem. 1995;41(10): $1414-1420$

10. American College of Sports Medicine. ACSM's Guidelines for Exercise Testing and Prescription. 9th ed. Philadelphia, PA: Lippincott Williams \& Wilkins; 2014.

11. Houmard JA, Tanner CJ, Slentz CA, Duscha BD, McCartney JS, Kraus WE. Effect of the volume and intensity of exercise training on insulin sensitivity. J Appl Physiol (1985). 2004;96(1):101-106.

12. Kraus WE, Torgan CE, Duscha BD, et al. Studies of a targeted risk reduction intervention through defined exercise (STRRIDE). Med Sci Sports Exerc. 2001;33(10):1774-1784.

13. Slentz CA, Duscha BD, Johnson JL, et al. Effects of the amount of exercise on body weight, body composition, and measures of central obesity: STRRIDE - a randomized controlled study. Arch Intern Med. 2004;164(1):31-39.

14. Grundy SM, Brewer HB Jr, Cleeman JI, et al. Definition of metabolic syndrome: Report of the National Heart, Lung, and Blood Institute/ American Heart Association conference on scientific issues related to definition. Circulation. 2004;109(3):433-438.

15. Alberti KG, Eckel RH, Grundy SM, et al. Harmonizing the metabolic syndrome: A joint interim statement of the International Diabetes Federation task force on epidemiology and prevention; National Heart, Lung, and Blood Institute; American Heart Association; World Heart Federation; International Atherosclerosis Society; and International Association for the Study of Obesity. Circulation. 2009;120(16): $1640-1645$

16. D'Agostino RB Sr, Vasan RS, Pencina MJ, et al. General cardiovascular risk profile for use in primary care: the Framingham Heart Study. Circulation. 2008;117(6):743-753.

17. Sackett DL, Rosenberg WM, Gray JA, Haynes RB, Richardson WS. Evidence based medicine: what it is and what it isn't. BMJ. 1996; 312(7023):71-72.

18. Amonette WE, English KL, Ottenbacher KJ. Nullius in Verba: A call for the incorporation of evidence-based practice into the discipline of exercise science. Sports Med. 2010;40(6):449-457.

19. Bouchard C, Rankinen T. Individual differences in response to regular physical activity. Med Sci Sports Exerc. 2001;33(6 Suppl): S446-S451.

20. Dunn AL, Marcus BH, Kamper JB, Garcia ME, Kohl HW 3rd, Blair SN. Comparison of lifestyle and structured interventions to increase physical activity and cardiorespiratory fitness: A randomized trial. JAMA. 1999;281(4):327-334.

\section{Publish your work in this journal}

Diabetes, Metabolic Syndrome and Obesity: Targets and Therapy is an international, peer-reviewed open-access journal committed to the rapid publication of the latest laboratory and clinical findings in the fields of diabetes, metabolic syndrome and obesity research. Original research, review, case reports, hypothesis formation, expert opinion and commentaries are all considered for publication. The manuscript management system is completely online and includes a very quick and fair peer-review system, which is all easy to use. Visit http://www.dovepress.com/testimonials.php to read real quotes from published authors. 